\title{
Controlling the Wettability and Adhesion of Carbon Fibers with Polymer Interfaces via Grafted Nanofibers
}

\author{
Seokjin Hong ${ }^{1}$, Majid Minary-Jolandan ${ }^{3}$, and Mohammad Naraghi ${ }^{1,2,4}$
}

\author{
${ }^{1}$ Department of Materials Science and Engineering, Texas A\&M University, College Station, \\ Texas 77843, United States, \\ 2 Department of Aerospace Engineering, Texas A\&M University, College Station, Texas \\ 77843, United States, and \\ ${ }^{3}$ Department of Mechanical Engineering and UT Dallas NanoTech Institute, University of \\ Texas at Dallas, Richardson, Texas 75080, United States. \\ ${ }^{4}$ corresponding author, naraghi@aero.tamu.edu
}

\begin{abstract}
Interfacial properties in carbon fiber composites is one of the key parameters controlling their structural functionality. Here, we introduce a novel method to engineer carbon fiberepoxy interfaces, via inclusion of nanofibers, towards higher interfacial strength and energy dissipation. In our method, stabilized polyacrylonitrile (PAN) nanofibers are grafted onto carbon fibers via electro-spinning process, followed by nanofiber consolidation via solvent vapor and thermal treatment. These treatments partially dissolves nanofibers along the nanofiber-fiber interface and trigger entropic elasticity in nanofibers, thus, increasing the nanofiber-fiber interactions. The hybridization of carbon fibers with PAN nanofibers increased the interfacial shear strength (IFSS) by $\sim 48 \%$, from $10.8 \pm 2.6$ to $15.9 \pm 4.9 \mathrm{MPa}$. Postmortem fractography points to mechanical interlocking between nanofibers and epoxy and reinforcing effects of nanofibers in matrix as root causes of IFSS enhancement. As a result of adding nanofibers to carbon fiber, junction failure mode changes from a dominantly adhesive failure (at epoxy-fiber interface) to dominantly cohesive failure, and failure plane slightly shifts away from epoxy-fiber interface to within the epoxy. Compared to other types of whiskers grown on carbon fibers, such as CNTs, the method proposed here requires low temperatures (below $300^{\circ} \mathrm{C}$ ), during which no surface damages are expected to accumulate on carbon fibers.
\end{abstract}

Keywords: Hybrid composites, Polymer-matrix composites (PMCs), Interface, Electrospinning, Fiber/matrix bond

Page 1 


\section{INTRODUCTION}

Over the past few decades, carbon fibers have been extensively used as reinforcements of polymer matrix composites in many high-value industries, such as automotive and aviation, sporting goods and military applications. For these industries, carbon fibers offer excellent mechanical properties at reduced weight, high thermal and electrical conductivities [1]. However, in addition to fiber strength, it is widely accepted that the mechanical properties of carbon fiber reinforced composites, such as in-plane and inter-laminar strength, longitudinal and transverse strength and fracture toughness, directly correlate with interfacial shear strength (IFSS) between the fiber and matrix [2-6]. On the other hand, as-carbonized fibers typically have defective surfaces, which is due to high furnace temperatures experienced during carbonization $\left(>800^{\circ} \mathrm{C}\right)[6,7]$. As a result of this high temperature, carbon fibers are coated with atomically thin layers of polycyclic hydrocarbons [8]. The surface defects and hydrocarbons act as week bonding sites and lubricants between fiber and matrix, leading to poor fiber-matrix interactions, characterized by IFSS of as low as $20-60 \mathrm{MPa}[6,7,9-11]$.

To enhance fiber-matrix interactions, several approaches have been proposed and implemented, many of which have been extensively used in industrial scale. For example, a common practice in aerospace industry is oxidative treatment of carbon fiber surfaces, by which weakly bonded coatings are removed and fiber surfaces are chemically functionalized [12]. The functional groups will form chemical/physical bonds with epoxy matrices during the epoxy curing process, leading to improved IFSS $[6,7,10,11]$. In addition, the oxidative treatment of the fiber surfaces can improve the surface roughness of the fiber, and lead to improvements in IFSS via mechanical interlocking [11].

Another approach to enhance fiber-matrix interactions is to grow or attach nanoscale 
whiskers with high aspect ratio and surface to volume ratio onto the surface of carbon fibers. The nanoscale whiskers substantially increases the load transfer area between the fibers and the matrix, leading to an enhancement in fiber-matrix interaction and effective IFSS [13]. The composites reinforced with whiskerized (fuzzy) fibers may also be referred to as "hybrid composites", pointing to the hierarchy in length scale of reinforcements $[14,15]$. Recently and in the past decade or so, the research on hybrid composites has taken a new turn by the introduction of carbon nanotube (CNT) and carbon nanofiber (CNF) coated carbon fibers. Several studies have investigated the chemical vapor deposition (CVD) of CNTs on carbon fibers, in which carbon fibers serve as the substrate [16-20]. Through single fiber pull out experiments, An et al., demonstrated that the CVD growth of CNTs on carbon fibers can improve IFSS by $\sim 100 \%$, from $65 \mathrm{MPa}$ to $126 \mathrm{MPa}$. It was demonstrated that the CNTs served as anchors inside the matrix, improving the load transfer between the fiber and the matrix. The improvement in IFSS was partly as a result of a dramatic increase in the loadtransfer area between them [20]. However, the CVD growth of CNTs on carbon fibers may lead to thermal degradation of the carbon fibers and catalyst diffusion inside carbon fibers, mainly due to high temperatures required for $\mathrm{CNT}$ growth, which will result in a considerable loss in mechanical strength of fibers $[16,18,20]$. To minimize carbon fiber thermal degradation, alternative recipes of $\mathrm{CNT}$ growth at temperatures as low as $500^{\circ} \mathrm{C}$ have been carried out, with minimal damage to carbon fibers [21]. However, the CNTs formed at relatively low temperatures often are highly defective, and as such, the application of this method to improve IFSS is limited. To avoid fiber thermal degradation while maintaining high quality of CNTs, Garcia et al [22] transferred forests of CVF grown CNTs to prepreg carbon fibers at room temperature, which led to $>100 \%$ increase in mode I and II fracture toughness. 
An alternative method to develop hybrid fibers is electrophoresis (EP). This method allows for the deposition of a wide range of nanoscale reinforcements, such as CNTs, CNFs, and graphite nanoparticles on carbon fibers [23-25]. Even though significant improvement in the fiber-matrix IFSS has been reported via this method, the exposure of the carbon fiber to an electric field in the electrophoresis solution led to damage in the fiber surface, which resulted in a 15-40\% reduction in fiber strength depending on the initial condition of the fiber, such as the surface chemistry of the fibers and the duration of electrophoresis [24].

Therefore, current whiskerization methods are faced with several challenges, such as carbon fiber degradation, lack of controllability over mechanical properties of whiskers and the whisker-carbon interactions. Moreover, CNTs are often cost prohibitive for most composite applications. To overcome these challenges, we propose a new concept for hybrid composites, based on depositing thermally stable polymeric nanofibers on carbon fibers, in which the nanofiber-fiber interactions is controlled via exposure of nanofibers to vapor of the polymer solvent and temperatures below $300^{\circ} \mathrm{C}$, to avoid thermal degradation of carbon fibers. The polymeric nature of the nanofibers is expected to enhance the energy dissipation at the fiber-matrix interface, leading to strong and tough junctions. The single fiber pull out tests revealed $\sim 48 \%$ improvement on the IFSS between the fiber and epoxy matric, as a result of a combination of mechanical interlocking and physical interactions between deposited nanofibers and matrix.

\section{EXPERIMENTAL SECTION}

Single carbon fibers (SGP203CSDL), with average diameter of $\sim 5.7 \mu \mathrm{m}$ were purchased from Hexcel Corporation. Polyacrylonitrile (PAN) $(\mathrm{MW}=150,000)$ was purchased in powder form from Sigma-Aldrich. Dimethylformamide (DMF), purchased from Sigma-Aldrich, was 
used as the solvent to dissolve the PAN powder for nanofiber production via electrospinning process. Epoxy resin and curing agent, (EPON 826 and EPIKURE W, respectively), were purchased from Miller-Stephenson Chemical Company, Inc., and were used to make epoxy micro-droplets on carbon fibers.

Fabrication of Hybridized Carbon Fiber. Processing of hybridized carbon fibers was conducted in three steps - all in air- as shown in Figure 1. In the first step, a single carbon fiber was fixed onto a copper frame, and the copper frame was placed and secured on the electrospinning drum collector. Then, a solution of PAN with a concentration of $9 \mathrm{wt} \%$ was electrospun onto the single carbon fiber for 30 seconds, at a temperature of $25^{\circ} \mathrm{C}$ and relative humidity of $20 \%$. To ensure full coverage of the fiber surface, the solution of PAN was also electrospun onto the other side of the single carbon fiber for the same duration. For electrospinning, the flow rate of the solution, voltage, and the distance between the tip of the needle and collector were $0.5 \mathrm{ml} / \mathrm{h}, 16 \mathrm{kV}$ and $200 \mathrm{~mm}$, respectively.

Scanning electron microscope (SEM) images of the grafted carbon fibers revealed that the majority of the nanofibers at this stage were laid parallel to the carbon fiber, and loosely attached to it. Therefore, in step 2, the carbon fibers which were coated with electrospun PAN nanofibers were exposed to DMF vapor to partially dissolve PAN and the nanofiber-fiber interface, thus increase the contact area and interactions between carbon fibers and the nanofibers. To this end, carbon fibers with deposited nanofibers were placed into a closed chamber filled with DMF vapor. The saturated environment of DMF vapor was generated by placing the DMF container on a hot plate at a temperature of $60^{\circ} \mathrm{C}$ inside the chamber, as shown in Figure 1 (step2).

Lastly (step 3), the PAN nanofibers electrospun on single carbon fibers were thermally Page 5 
stabilized in air, by exposing the carbon fiber to temperatures of as high as $265^{\circ} \mathrm{C}$ for 2 hours in an oven. During this process, several reactions take place in PAN nanofibers, including cyclization and de-hydrogenation, converting the molecular structure of the linear PAN into the ladder-like and more stable structure [26]. This step is critical in processing of hybrid fibers, since the as-fabricated PAN, unlike the stabilized PAN, will generally get dissolved in epoxy during the curing process (at temperatures of $\sim 150-175^{\circ} \mathrm{C}$ ).

Single Fiber Pull-Out Tests. To evaluate the IFSS of carbon fibers and Epon 862 epoxy, single fiber pull out tests were carried out. Epoxy micro-droplets were placed on single bare and hybrid carbon fibers. The epoxy was made by mixing resin/curing agent with a weight ratio of 1 to 0.265 . The droplets, in liquid form and prior to curing, were placed on fibers by using a needle with a fine tip. The epoxy micro-droplets were cured for 2 hours at $125^{\circ} \mathrm{C}$ and then for 2 hours at $175^{\circ} \mathrm{C}$ inside an oven. The diameter of the epoxy microdroplets ranged from about $45 \mu \mathrm{m}$ to about $258 \mu \mathrm{m}$, fairly controllable based on the initial (prior to curing) size of the droplet. Examples of carbon fibers, partially embedded in cured epoxy droplets, are shown in Figure 2 (a) and (b).

The pull out tests was performed on a Gatan MT10365 tensile testing device with modified grips. For this purpose, the carbon fiber, with the epoxy droplet, was placed on the device, with its one end fixed to the load cell, while the epoxy micro-droplets was carefully placed across a channel with the width of $\sim 20 \mu \mathrm{m}$. This width was sufficiently wider than the fiber diameter and narrower than the diameter of the droplet, to allow for friction free sliding of the fiber, while blocking the motion of the droplet when the device is actuated, thus loading the fiber-matrix interface in shear (Figure 2 (c)).

The single fiber pull-out test was carried out with a cross-head speed of $2 \mu \mathrm{m} / \mathrm{min}$. The 
average value of the interfacial shear strength (IFSS) $\tau_{I F S S}$ was estimated as:

$$
\tau_{I F S S}=\frac{F_{\max }}{\pi d l}
$$

where $F_{\max }, d$, and $l$ are the junction load capacity (the maximum load on the fiber and the junction, at which the failure was initiated), diameter of single carbon fibers, and the embedded length of the fibers in epoxy droplet, respectively. A minimum of 13 experiments were performed for each type of fibers (bare or hybridized).

Effect of exposure to DMF and heat treatment on carbon fiber surface and sizing. To further investigate the possibility of sizing removal and accumulation of surface damages on carbon fibers as a result of DMF vapor and heat treatments, we exposed a few bare carbon fibers to $265^{\circ} \mathrm{C}$, followed by DMF vapor treatment, similar to the protocol that is used to coat carbon fibers with PAN nanofibers. The treated bare carbon fibers were then subjected to pull out tests and wetting angle measurements.

Morphology of bare/hybridized Carbon Fibers and Fracture surface. To shed light on the interfacial load bearing and energy dissipation mechanisms, the surface morphologies of bare and hybridized carbon fibers, and the interfacial fracture surface between the carbon fibers and the epoxy were characterized via SEM (FEI QUANTA 600 FE-SEM).

\section{RESULT AND DISCUSSION}

Surface morphology of carbon fibers. SEM images of bare carbon fibers, and hybridized carbon fibers before and after solvent treatment, revealed the evolution of the fiber surface morphology during the hybridization process. As shown in Figure 3(a), bare single carbon fibers have an average diameter of about $5.7 \mu \mathrm{m}$ with smooth surfaces. In addition, as 
shown in Figure 3(b), the as-electrospun nanofibers are mainly aligned with the axis of the fibers, because of electrostatic attraction between the fibers and the nanofibers. On the other hand, it is evident in the figure that the majority of nanofibers are not directly connected to the carbon fiber. This arrangement of nanofibers is not likely to contribute to fiber matrix interactions, due to poor fiber-nanofiber interactions.

To overcome this problem, the carbon fibers which were coated with PAN nanofibers were exposed to DMF vapor, as described in the Experimental Section. The DMF vapor was intended to condense on surface of the carbon fiber and partially dissolve PAN nanofibers at the nanofiber-fiber interface, leading to increased contact area between the nanofibers and the fiber. The success of this approach in enhancing the interactions between nanofibers and the host fiber is evident, as nanofibers become conformal to the surfaces of fibers as a result of exposure to DMF (Figure 3(c)). The hybridized fibers were heat treated at $265^{\circ} \mathrm{C}$ for 2 to enhance thermal stability of PAN nanofibers in epoxy, during the curing process, as discussed in the Experimental Section.

Surface wettability of Carbon Fibers. The contact angle of the epoxy microdroplets embedded with bare and hybridized carbon fiber was measured via optical microscopy as shown in Figure 4. The hybridized carbon fiber/epoxy micro-droplet composite has a markedly lower contact angle than bare carbon fiber/epoxy micro-droplet composite, indicating a higher wettability between hybridized carbon fiber and the matrix. The higher wettability of hybridized fibers suggests an affinity between stabilized PAN and epoxy. Moreover, the increased surface roughness of the hybridized carbon fibers, compared to bare fibers, is partly responsible for the enhanced wettability of the former, as the interface between liquid epoxy (prior to curing) will follow the rougher surface of hybridized fibers to 
minimize the surface energy [27].

Single Carbon Fiber Pull-Out Experiment. Pull-out experiments were carried out to evaluate the effect of carbon fiber hybridization on interfacial shear strength between fiber and epoxy matrix. During each pull out test, the force applied on the junction was increased until the junction failed. The force on the fiber, which is equal to the total force carried by the fiber-matrix interface (force equilibrium of carbon fiber), was recorded as a function of the cross head displacement. An example of the force $v s$. cross head displacement for the two cases of bare and hybridized carbon fibers is shown in Figure 5(a) and (b), respectively. As shown in the figure, the force initially increases linearly in both types of fibers, until the force capacity of the junction is reached and the junction fails. However, the post-peak mechanical behavior of the junctions is markedly different between the two types of fibers. In the case of bare fibers, the junction failure was catastrophic, and subsequent to reaching the peak force, the junction lost nearly its entire load bearing capacity. In contrast, in hybrid fibers, the junction failure is not catastrophic. Instead, subsequent to reaching the peak load, junction retained a considerable portion of its load bearing capacity, with significant energy being dissipated in the post-peak sliding. More interestingly, in a few hybrid fibers, a second force peak, higher than the first peak was observed. This post-peak residual load capacity is likely caused by mechanical interlocking at the interface of nanofiber coated fibers and epoxy droplet. Therefore, our proposed approach for carbon fiber hybridization offers great potential to enhance both junction strength (load bearing capacity) and toughness (post-peak energy dissipation). However, it is to be pointed out that more research is needed here to characterize the post-peak behavior, as this residual load capacity showed a considerable scatter between different samples, likely due to variations in surface roughness of hybridized nanofibers along their length. 
To investigate the effect of carbon fiber hybridization on load bearing capacity of junctions, the pull out tests were interpreted in terms of effective IFSS, calculated based on Equation (1).

\section{As shown in}

Figure 6, hybridization of carbon fibers with thermally stabilized PAN nanofibers increased the IFSS, on average by $\sim 48 \%$, from $10.8 \pm 2.6 \mathrm{MPa}$ to $15.9 \pm 4.9 \mathrm{MPa}$. The uncertainties in the measurements are the standard deviations of a minimum of 13 measurements. Higher uncertainty in the IFSS of hybridized fibers is potentially rooted in non-uniformities in the nanofiber coatings between samples and/or along each sample.

In addition to the comparisons made between IFSS of hybridized carbon fibers and asreceived bare fibers, to investigate the possibility of sizing removal and accumulation of surface damages on carbon fibers as a result of DMF vapor and heat treatments, we exposed a few bare carbon fibers to $265^{\circ} \mathrm{C}$ and DMF vapor, following the protocol that was used to coat carbon fibers with PAN nanofibers. The treated bare carbon fibers revealed an IFSS of 9.9 $\pm 2.2 \mathrm{MPa}$ and wetting angle of $70.2^{\circ}$ with the epoxy, very close to the corresponding values of the as-received (untreated) bare carbon fibers, $10.8 \pm 2.6 \mathrm{MPa}$ and $73.4^{\circ}$, respectively. Hence, the adhesion and interactions between the epoxy and the carbon fibers -in the absence of nanofibers - is not influenced by treatments. As such, it is concluded that surface of the carbon fiber remains unchanged, ruling out the possibility of sizing removal. Moreover, since the sizing removal is the prelude to the accumulation of damage on carbon fibers, the possibility of the latter is also excluded.

More insight into the origin of IFSS enhancement via nanofiber hybridization was obtained by postmortem SEM imaging of pulled out fibers and their epoxy holes. Typical images of fibers and holes for both bare and hybridized fibers are shown side by side in 
Figure 7. Post mortem images of bare carbon fibers points to a dominantly adhesive junction failure, along the fiber-matrix interface, evident from the clean surfaces of the pulled out fibers (no or insignificant epoxy residues). For instance, the portions of the fiber shown with rectangles (1) and (2) in Figure 7(a), and their zoomed out views in Figure 7 (b), point to a dominantly clean fiber surface after pull out (rupture along the fiber-epoxy interface). The boundaries of the epoxy droplet prior to pull out are shown with broken lines in Figure 7(a) for clarity. It is to be noted that cohesive failure (within the matrix) of the bare fiber-epoxy junction along a surface parallel to the fiber surface was also observed with lower frequency than adhesive failure, as it is evident from the epoxy residues in some portions of the fiber, e.g., shown in Figure 7 (b).

In contrast to bare fibers, post failure SEM images of the hybridized fibers, along the initial embedded length, suggests that their junction failure is mostly cohesive. The epoxy residues on the pulled out fibers, shown in Figure 7(d) and (e), are indications of cohesive failure of the junction within the epoxy near the fiber-epoxy interface. In addition, rare cases of adhesive failure were also observed, such as the portions of the fiber with no apparent epoxy residue in rectangle 2 of Figure 7(d) and the zoomed out view in Figure 7(e). These cases have likely occurred at locations where the local density of the PAN nanofibers was low, as this type of failure is similar to dominant failure mode of bare fibers. In addition, the diameter of epoxy holes for the case of bare fiber pull out is close to the fiber diameter, while for hybridized fibers, the holes are slightly larger than the diameter of the bare fibers, consistent with the adhesive and cohesive junction failures of bare and hybridized fibers, respectively.

Moreover, a comparison between the surface morphology of the epoxy holes for the two 
cases of fibers reveals that the postmortem surfaces of the holes have a considerably higher roughness in case of hybridized fibers compared to bare ones (Figure 7 (c) \& (f)). The rough surfaces of the former can be explained based on mechanical interactions between the epoxy and PAN nanofibers, such as reinforcing effect of the latter, which prevented the growth of the interfacial debonding along the fiber surface, consistent with the predominantly cohesive junction failure of epoxy-hybridized fibers (Figure 7 (e)).

Assuming that the cohesive failure leading to pull out is stress-driven, higher roughness of fracture surface in the epoxy droplet of hybrid fibers compared to bare ones, suggests a more complex state of stress with considerable local variations of the direction of principal stresses along the fiber surface. The source of local stress variations can be the inhomogeneity of the matrix surrounding the hybrid fibers, due to reinforcing effect of nanofibers and mechanical interlocking between the epoxy and matrix. This effect can be inferred from the sub-micron diameter holes, observed in the SEM images of the epoxy coatings of pulled out hybrid fibers (Figure 7(e)). These holes are likely where individual nanofibers are pulled out of the epoxy during carbon fiber during junction failure. In other words, prior to nanofiber pull out, the nanofibers were carrying axial load and thus reinforcing the matrix. Therefore, because of reinforcing effect of nanofibers and mechanical interlocking between them and epoxy, the "weakest plane", where the junction failure is most likely, is pushed further away from the fiber, leading to cohesive failure. Hence, the fracture surface is expanded proportional to that, demanding a larger force to initiate junction failure. The larger junction load capacity ( 48\%) manifests itself as enhanced IFSS (Figure 6). This failure mechanism is schematically shown in Figure 8.

The proposed cohesive failure in epoxy-hybrid fiber junctions requires sufficiently strong 
bonding between PAN nanofibers and carbon fibers, without which a propagating interface crack could cause debonding between PAN and carbon fiber (adhesive failure), a scenario which was rarely observed (Figure 7 (b)). The origin of this sufficiently strong bond, although expected to be primarily via physical bonds such as vdW forces, is likely rooted in two causes. First, as mentioned in the Experimental Section, the DMF vapor condensates on carbon fiber surface, partially dissolving the PAN nanofibers near the fiber surface. The surface tension between DMF and PAN, and also DMF and carbon fiber, as the DMF is being vaporized during PAN thermal stabilization, causes the nanofibers to collapse on the carbon fiber. As a result, the nanofibers become conformal to fiber surface. The second cause is rooted in the molecular alignment and crystallinity of electrospun PAN nanofibers [28]. In other words, as electrospun PAN nanofibers contain aligned chains that are partially aligned with nanofiber axis, caused by electrostatic stretching during electrospinning process. Due to chain alignment and order in chain arrangement, the entropy of the as electrospun PAN nanofibers are less than the maximum entropy which can be achieved in the case of random alignment. This chain alignment is stable at room temperature due to low solvent content of the as electrospun nanofibers [28]. However, the diffusion of DMF to PAN nanofibers (step 2 in Figure 1) which had been deposited on carbon fibers acts as a plasticizer in PAN, increasing chain mobility. The chain mobility will be further enhanced during thermal stabilization (step 3, Figure 1), as the temperature of PAN is raised above its $\operatorname{Tg}\left(\sim 90^{\circ} \mathrm{C}\right)$. The enhanced chain mobility will inherently relax the aligned chains and deform the fiber towards maximum entropy. Hence, chains retract and the nanofibers length will shrink. As a result, the PAN nanofibers which are partly conformal to fiber surface due to DMF surface tension will tightly wrap around the carbon fiber, further enhancing fiber-nanofiber interaction.

To demonstrate the significance of this length change, we exposed free standing Page 13 
electrospun ribbons of PAN to saturated environment of DMF solvent, followed by thermal stabilization, and monitored their length change. The steps were similar to those applied to PAN nanofiber coatings of hybridized carbon fibers, as shown in Figure 1. The optical images of the PAN ribbon before and after solvent treatment and thermal stabilization is shown in Figure 9 (a), pointing to a considerable change in length after each of the two steps. As shown in Figure 9(b), the length of the ribbon of nanofibers reduced by $\sim 20 \%$ after solvent vapor treatment, and the total change in length nearly tripled after thermal stabilization. The change in length is calculated relative to the final length of the ribbons as the reference length. This change in length, for the nanofibers that are conformal to the fiber surface (for instance via surface tension), will tighten the nanofibers around the carbon fiber, leading to enhanced friction and load transfer between them.

\section{CONCLUSION}

The fabrication of the hybridized carbon fiber was attained by electrospinning PAN solutions onto individual carbon fibers. Pull out tests revealed a $\sim 48 \%$ improvement in the effective IFSS as a result of adding nanofibers to fiber surface. Postmortem fractography revealed a change in junction failure mode from adhesive failure of bare fiber-epoxy junctions (epoxy-fiber debonding) to cohesive failure of the hybridized fiber-epoxy junctions. The predominantly cohesive failure of the hybrid fiber-epoxy junctions requires strong bonding between the nanofibers and carbon fiber, strong enough to prevent debonding between them during failure. Our study suggests that this bonding is achieved via solvent vapor-based consolidation, which makes nanofibers conformal to the fibers surface, thus increasing the contact area between nanofibers and the fiber. Moreover, subsequent nanofiber shrinkage due to solvent effects and increase in temperature will trigger entropic chain recoiling. Compared to other whiskerization techniques of carbon fibers, such as CVD 
growth of CNTs on fibers, our technique requires significantly lower temperatures, thus, no thermal damages are expected to occur on carbon fibers.

\section{ACKNOWLEDGMENT}

MN and MMJ acknowledge the support from NSF by NSF-CMMI award numbers 1450110 and 1450107. MN also acknowledges the supports from TAMU, through startup funds. 


\section{FIGURES}

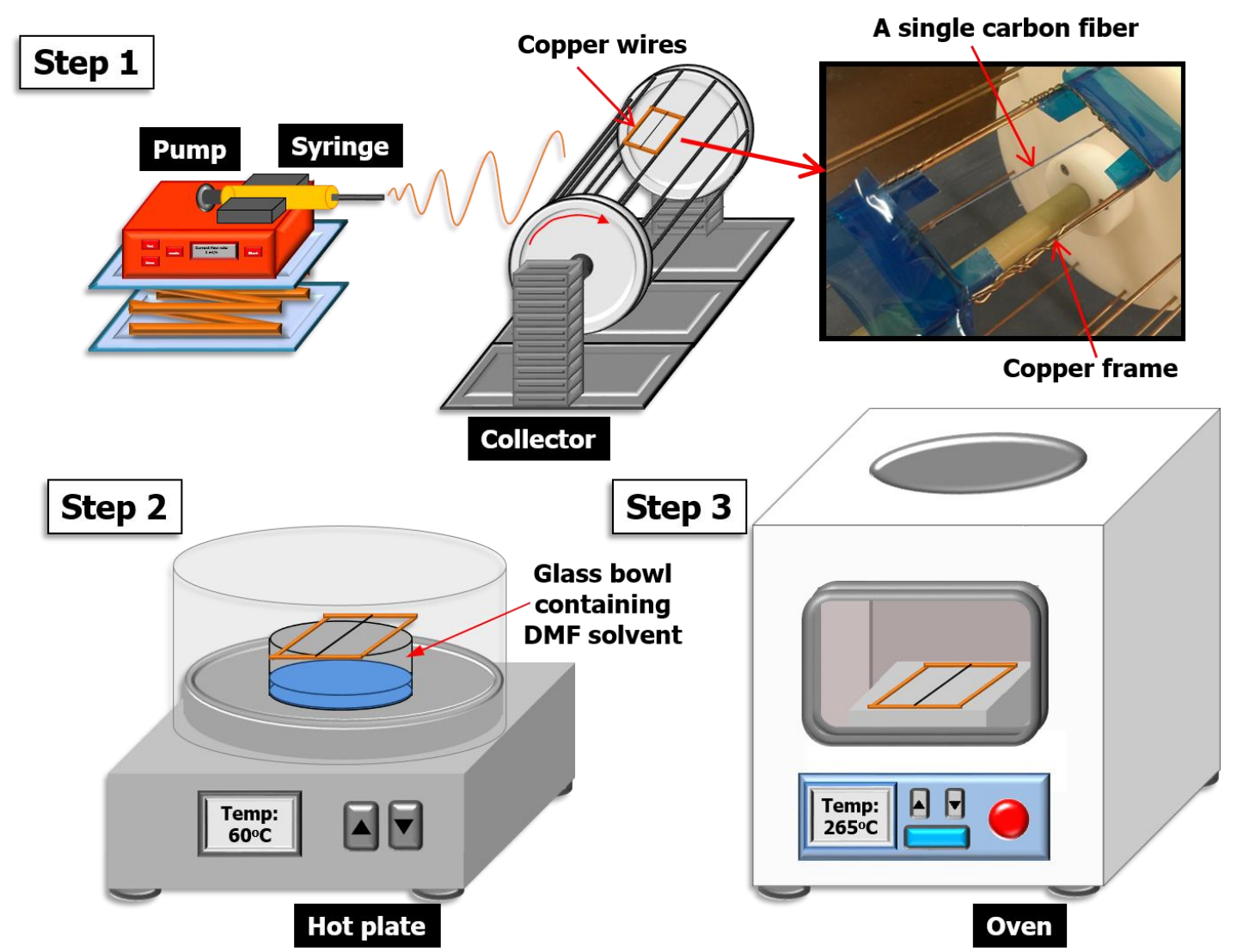

Figure 1. Schematics of steps for fabrication of hybridized carbon fibers: Step 1, electrospinning on single carbon fibers, step 2, exposure of a single carbon fiber which was grafted with electrospun PAN nanofibers to a vapor of DMF solvent, Step 3, thermal stabilization of PAN nanofibers electrospun on the carbon fiber at $265^{\circ} \mathrm{C}$. 

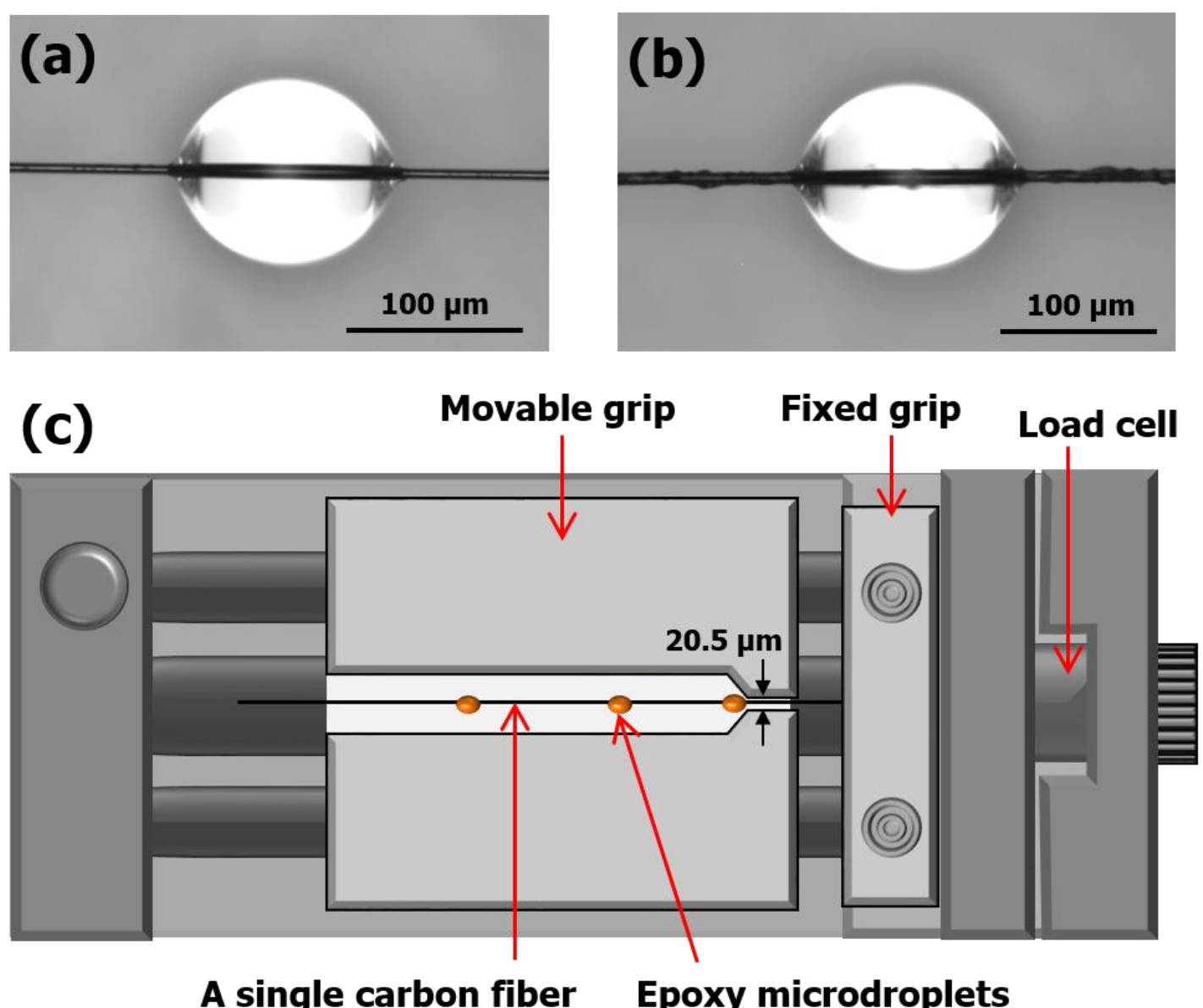

Figure 2. Optical microscope images of (a) an epoxy micro-droplet on a bare and (b) hybridized carbon fiber. (c) Schematic of the modified tensile tester machine to perform the pull out experiment. 


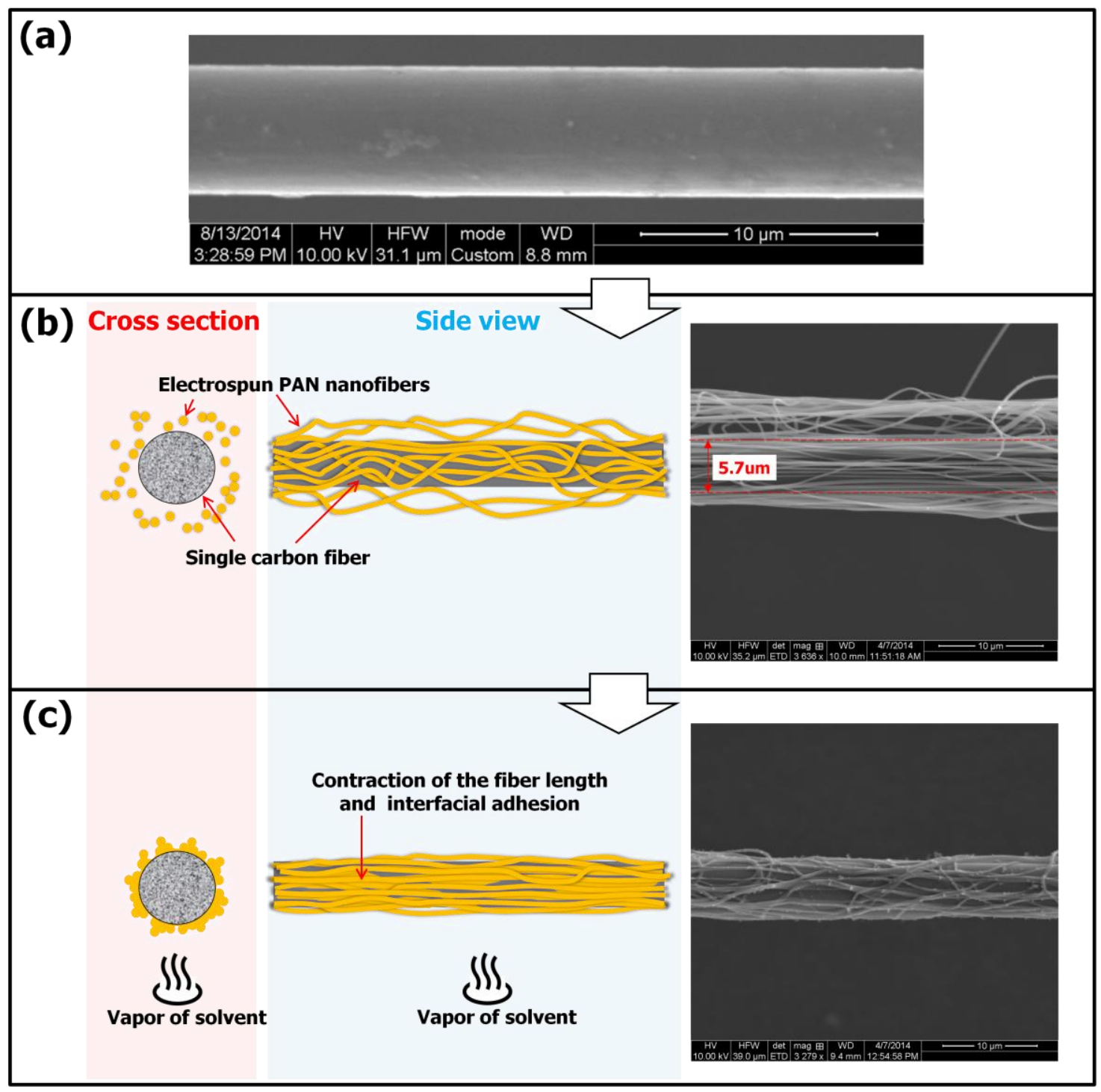

Figure 3. Schematic illustration and corresponding SEM images of the formation of hybridized carbon fibers. (a) Bare carbon fiber. (b) Carbon fibers coated with aselectrospun nanofibers. (c) Hybridized carbon fibers after solvent vapor treatment and thermal stabilization. 

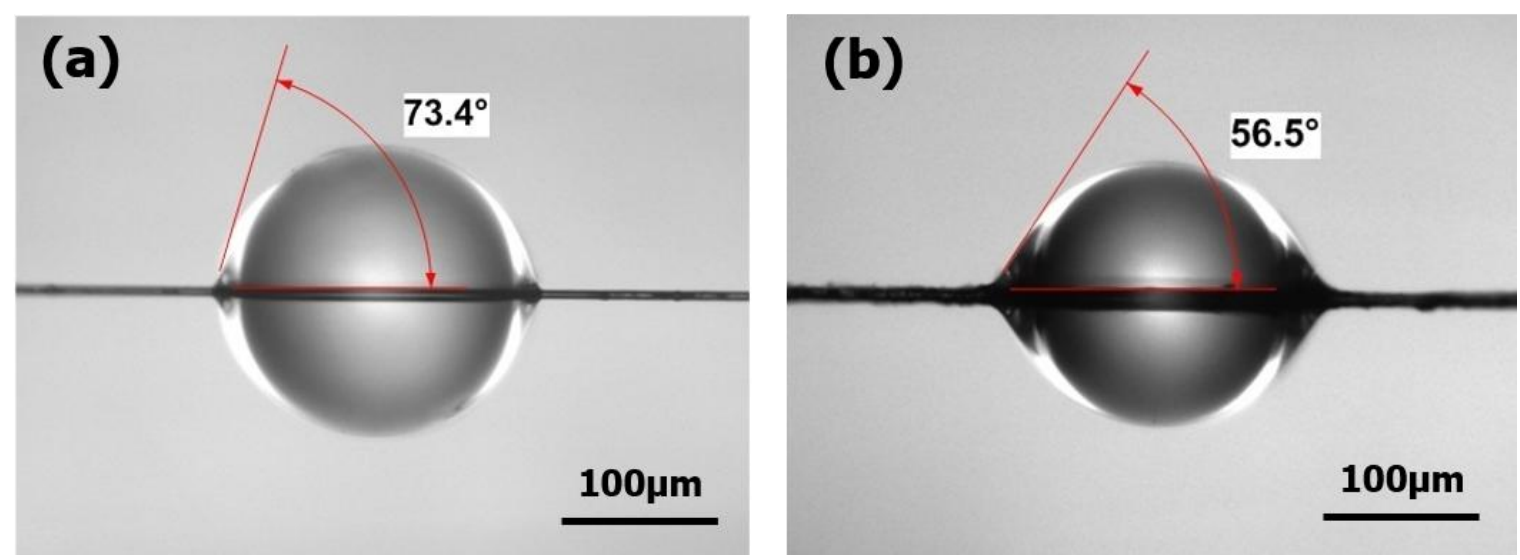

Figure 4. Contact angles between epoxy with (a) bare and (b) hybridized carbon fiber.
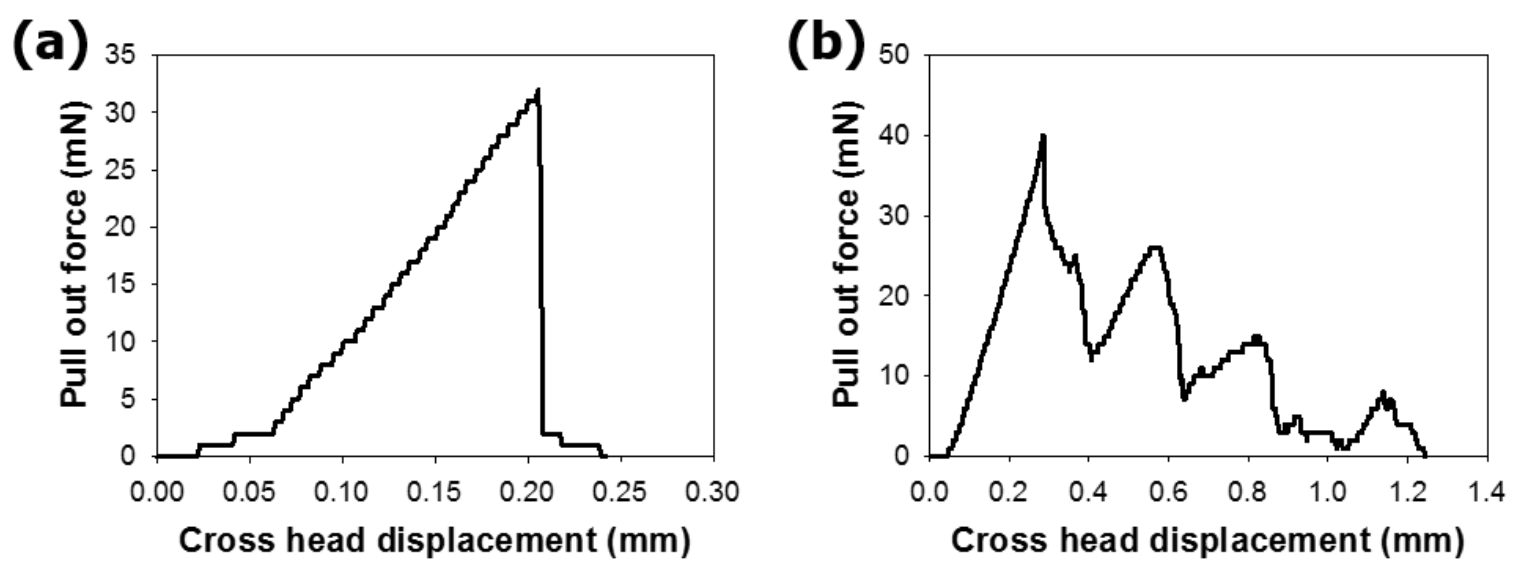

Figure 5. Pull out force vs. cross-head displacement curves of (a) bare and (b) hybridized carbon fibers from epoxy microdroplets. 


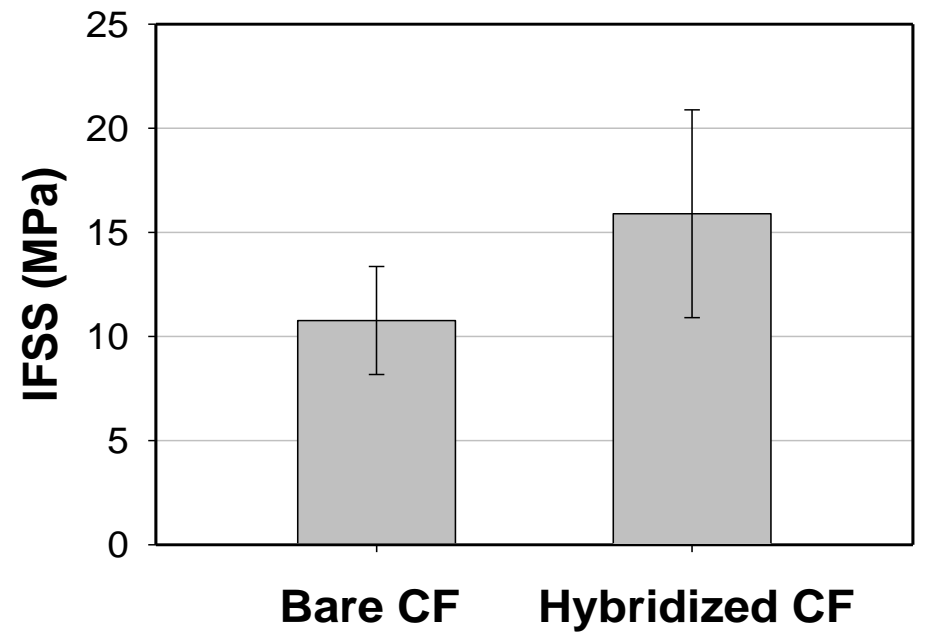

Figure 6. Comparison of IFSS of bare and hybridized carbon fiber/epoxy micro-droplet. 


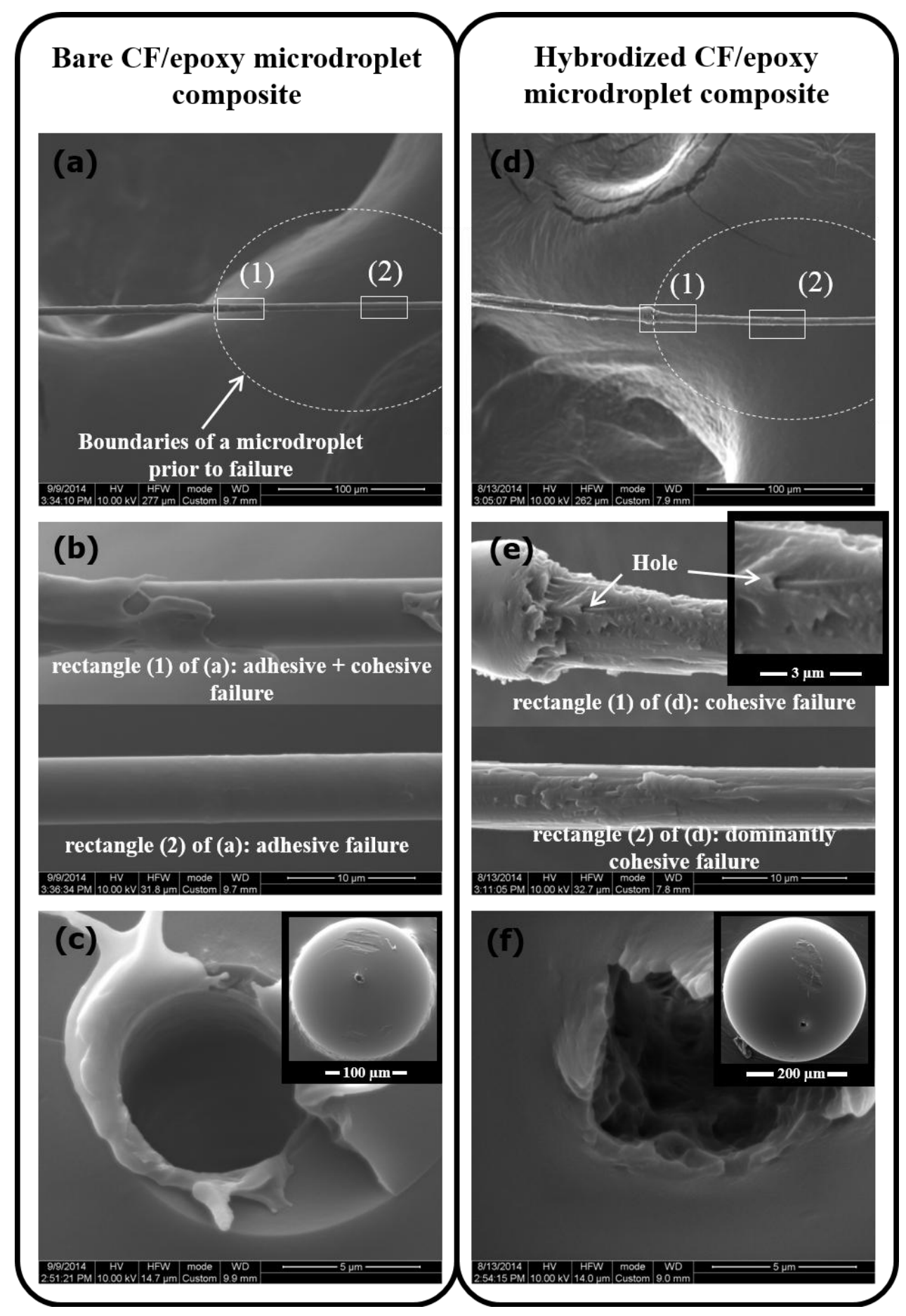

Figure 7. Postmortem SEM images of surface morphology of (a \& b) bare and (d \& e) hybridized carbon fibers. In (a) and (d), the boundaries of epoxy microdroplets prior to failure is shown with broken lines. The inner surface morphologies of the micro-droplet holes for the two cases of (c) bare and (f) hybridized fibers. The insets in (c) and (f) are SEM images of pulled out droplets. The pull out hole can be discerned in the inset. 


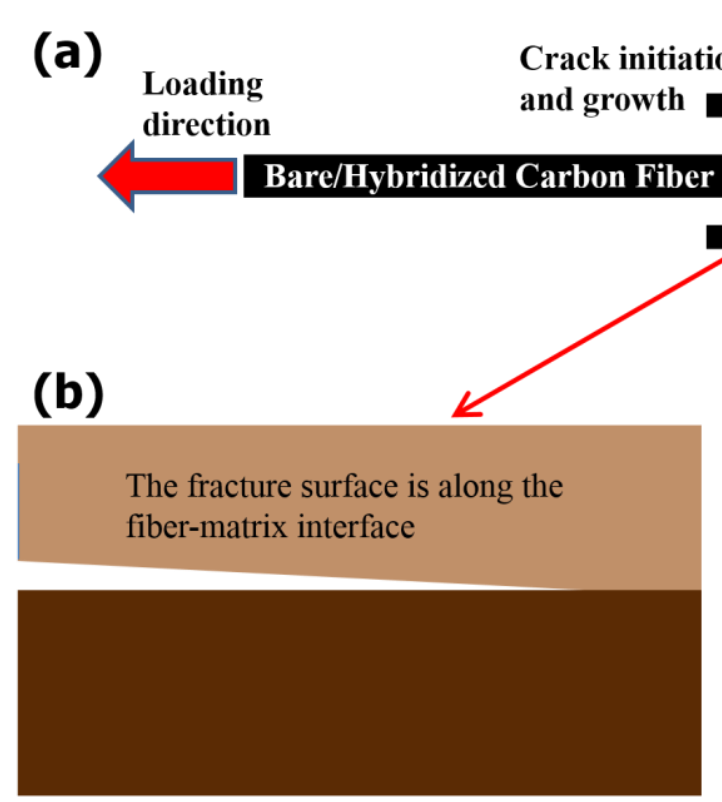

Bare carbon fiber (c)

Epoxy matrix

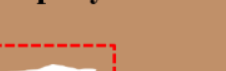


(a)

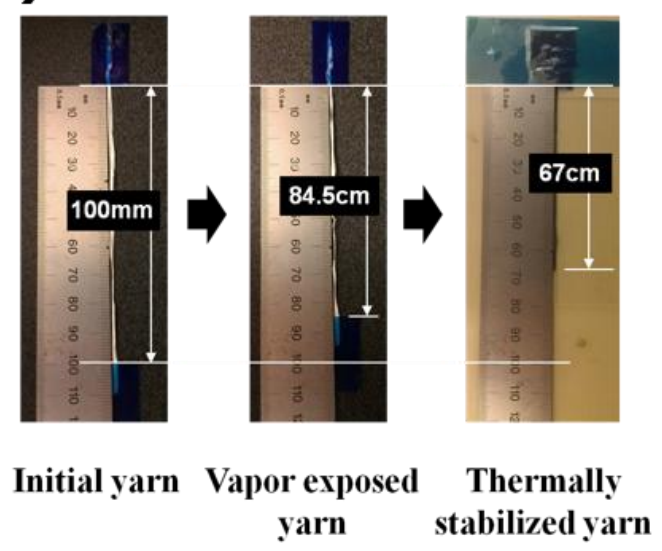

(b)

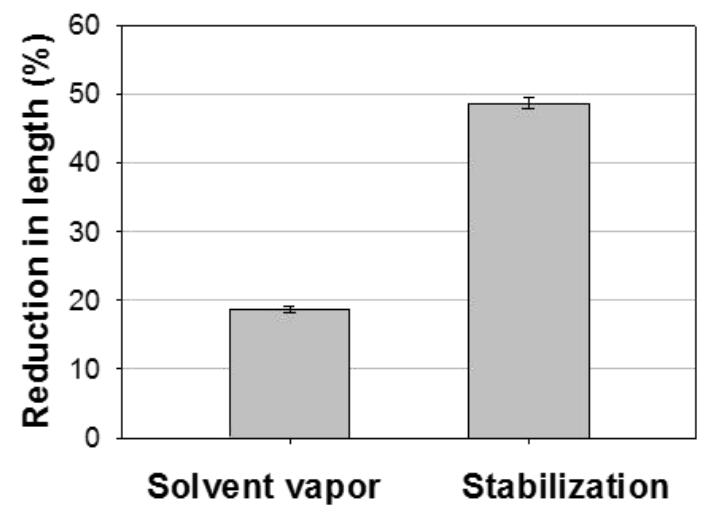

Figure 9. (a) Entropy driven shrinkage of electrospun PAN nanofiber yarns induced by solvent vapor and heat during stabilization. (b) Reduction in length normalized by the final length.

\section{REFERENCES}

1. Huang, X., Fabrication and Properties of Carbon Fibers. Materials, 2009. 2(4): p. 2369-2403.

2. Totry, E., Molina-Aldareguía, J.M., González, C., and Llorca, J., Effect of Fiber, Matrix and Interface Properties on the in-Plane Shear Deformation of Carbon-Fiber Reinforced Composites. Composites Science and Technology, 2010. 70(6): p. 970-980.

3. Madhukar, M.S. and Drazal, L.T., Fiber-Matrix Adhesion and Its Effect on Composite Mechanical Properties. I. Inplane and Interlaminar Shear Behavior of Graphite/Epoxy Composites. Journal of Composite Materials, 1991. 25(8): p. 932-957.

4. Madhukar, M.S. and Drzal, L.T., Fiber-Matrix Adhesion and Its Effect on Composite Mechanical Properties. Ii. Longitudinal $\left(0^{\circ}\right)$ and Transverse $\left(90^{\circ}\right)$ Tensile and Flexure Behavior of Graphite/Epoxy Composites. Journal of Composite Materials, 1991. 25(8): p. 958-991.

5. Madhukar, M.S. and Drazal, L.T., Fiber-Matrix Adhesion and Its Effect on Composite Mechanical Properties. Iii. Longitudinal $\left(0^{\circ}\right)$ Compressive Properties of Graphite/Epoxy Composites. Journal of Composite Materials, 1992. 26(3): p. 310-333.

6. Madhukar, M.S. and Drzal, L.T., Fiber-Matrix Adhesion and Its Effect on Composite Mechanical Properties: Iv. Mode I and Mode Ii Fracture Toughness of Graphite/Epoxy Composites. Journal of Composite Materials, 1992. 26(7): p. 936-968.

7. Drzal, L.T., Madhukar, M., and Waterbury, M.C., Adhesion to Carbon Fiber Surfaces: Surface Chemical and Energetic Effects. Composite Structures, 1994. 27(1-2): p. 6571. 
8. Macedo, F. and Ferreira, J.A., Thermal Contact Resistance Evaluation in PolymerBased Carbon Fiber Composites. Review of Scientific Instruments, 2003. 74(1): p. 828.

9. Raghavendran, V.K., Drzal, L.T., and Askeland, P., Effect of Surface Oxygen Content and Roughness on Interfacial Adhesion in Carbon Fiber-Polycarbonate Composites. Journal of Adhesion Science \& Technology, 2002. 16(10): p. 1283-1306.

10. Krekel, G., Zielke, U.J., Hüttinger, K.J., and Hoffman, W.P., The Relevance of the Surface Structure and Surface Chemistry of Carbon Fibres in Their Adhesion to High Temperature Thermoplastics. Journal of Materials Science, 1994. 29(15): p. 39843992.

11. Drzal, L.t., Drzal, N., and Sugiura, D., The Role of Chemical Bonding and Surface Topography in Adhesion between Carbon Fibers and Epoxy Matrices. Composite interfaces, 1996. 4(5): p. 337-354.

12. Li, J., Interfacial Studies on the Ozone and Air-Oxidation-Modified Carbon Fiber Reinforced Peek Composites. Surface and Interface Analysis, 2009. 41(4): p. 310-315.

13. Morgan, P., Carbon Fibers and Their Composites. 2005, Boca Raton: Taylor \& Francis.

14. Chen, Q., Zhang, L., Rahman, A., Zhou, Z., Wu, X.-F., and Fong, H., Hybrid MultiScale Epoxy Composite Made of Conventional Carbon Fiber Fabrics with Interlaminar Regions Containing Electrospun Carbon Nanofiber Mats. Composites Part A: Applied Science and Manufacturing, 2011. 42(12): p. 2036-2042.

15. Thostenson, E.T., Li, C., and Chou, T.-W., Nanocomposites in Context. Composites Science and Technology, 2005. 65(3-4): p. 491-516.

16. Qian, H., Bismarck, A., Greenhalgh, E.S., and Shaffer, M.S.P., Carbon Nanotube Grafted Carbon Fibres: A Study of Wetting and Fibre Fragmentation. Composites Part A: Applied Science and Manufacturing, 2010. 41(9): p. 1107-1114.

17. Thostenson, E.T., Li, W.Z., Wang, D.Z., Ren, Z.F., and Chou, T.W., Carbon Nanotube/Carbon Fiber Hybrid Multiscale Composites. Journal of Applied Physics, 2002. 91(9): p. 6034.

18. Sager, R.J., Klein, P.J., Lagoudas, D.C., Zhang, Q., Liu, J., Dai, L., and Baur, J.W., Effect of Carbon Nanotubes on the Interfacial Shear Strength of T650 Carbon Fiber in an Epoxy Matrix. Composites Science and Technology, 2009. 69(7-8): p. 898-904.

19. Mathur, R.B., Chatterjee, S., and Singh, B.P., Growth of Carbon Nanotubes on Carbon Fibre Substrates to Produce Hybrid/Phenolic Composites with Improved Mechanical Properties. Composites Science and Technology, 2008. 68(7-8): p. 16081615.

20. An, F., Lu, C., Li, Y., Guo, J., Lu, X., Lu, H., He, S., and Yang, Y., Preparation and Characterization of Carbon Nanotube-Hybridized Carbon Fiber to Reinforce Epoxy Composite. Materials \& Design, 2012. 33(0): p. 197-202.

21. De Greef, N., Magrez, A., Couteau, E., Locquet, J.-P., Forró, L., and Seo, J.W., Growth of Carbon Nanotubes on Carbon Fibers without Strength Degradation. physica status solidi (b), 2012. 249(12): p. 2420-2423.

22. Garcia, E.J., Wardle, B.L., and John Hart, A., Joining Prepreg Composite Interfaces with Aligned Carbon Nanotubes. Composites Part A: Applied Science and Manufacturing, 2008. 39(6): p. 1065-1070.

23. Schaefer, J.D., Rodriguez, A.J., Guzman, M.E., Lim, C.-S., and Minaie, B., Effects of Electrophoretically Deposited Carbon Nanofibers on the Interface of Single Carbon Fibers Embedded in Epoxy Matrix. Carbon, 2011. 49(8): p. 2750-2759. 
24. Park, J.K., Do, I.-H., Askeland, P., and Drzal, L.T., Electrodeposition of Exfoliated Graphite Nanoplatelets onto Carbon Fibers and Properties of Their Epoxy Composites. Composites Science and Technology, 2008. 68(7-8): p. 1734-1741.

25. Zhang, J., Zhuang, R., Liu, J., Mäder, E., Heinrich, G., and Gao, S., Functional Interphases with Multi-Walled Carbon Nanotubes in Glass Fibre/Epoxy Composites. Carbon, 2010. 48(8): p. 2273-2281.

26. Naraghi, M. and Chawla, S., Carbonized Micro- and Nanostructures: Can Downsizing Really Help? Materials, 2014. 7(5): p. 3820-3833.

27. Bico, J., Thiele, U., and Quéré, D., Wetting of Textured Surfaces. Colloids and Surfaces A: Physicochemical and Engineering Aspects, 2002. 206(1-3): p. 41-46.

28. Naraghi, M., Arshad, S.N., and Chasiotis, I., Molecular Orientation and Mechanical Property Size Effects in Electrospun Polyacrylonitrile Nanofibers. Polymer, 2011. 52(7): p. 1612-1618. 